\title{
Particularidades de las licenciaturas en humanidades y lengua castellana*
}

\author{
William Leonardo Perdomo Vanegas ${ }^{1}$ \\ Leidy Yohanna López Pineda ${ }^{2}$
}

Recibido: 20-05-2020

Aceptado: 05-07-2020

\section{Resumen}

El presente artículo tiene como objetivo socializar los resultados de un ejercicio investigativo, denominado "Caracterización de la formación de docentes en humanidades en Colombia", que tuvo como propósito explorar las tendencias y los referentes en torno a la formación de docentes en el área de las humanidades y lengua castellana en el país, con posterioridad al Decreto 2450 del 17 de diciembre de 2015 y a la Resolución 02041 de febrero de 2016. Para tal fin, la investigación se centró en un análisis descriptivo desde los criterios propuestos por las políticas nacionales en educación y la fundamentación conceptual sobre humanidades y lengua castellana. En ese orden de ideas, este artículo presenta un esbozo de las políticas educativas que permiten estructurar los currículos $y$, posteriormente, expone un análisis descriptivo de los programas de licenciatura en el área mencionada a partir de los conceptos sugeridos por la normatividad.

\footnotetext{
*Este artículo de investigación científica es resultado de un estudio desarrollado en el marco de la reforma curricular de las licenciaturas en la Facultad de Educación de la Corporación Universitaria Minuto de Dios.

1. Doctor en Literatura. Investigador en las líneas de investigación de lenguajes y procesos creativos y didácticas específicas de la Facultad de Educación de Uniminuto. Líder del grupo Ambientes de Aprendizaje.

ORCID: https://orcid.org/0000-0002-3979-7235

Google Scholar: https://scholar.google.es/citations?user=rRI1 xcQAAAAJ\&hl=es

Correo electrónico: wperdomo32@gmail.com
}

2. Licenciada en Humanidades y Lengua Castellana, maestrante en Estudios Culturales de la Universidad Nacional de Colombia.

ORCID: https://orcid.org/0000-0001-9708-9088

Google Scholar: https://scholar.google.com/citations?user=B5kxNUAAAAJ\&hl=es

Correo electrónico: llopezpi@unal.edu.co 
Palabras clave: formación de docentes, políticas educativas, humanidades, lengua castellana.

\title{
Particularities of the bachelor's degrees in humanities and Spanish language
}

\begin{abstract}
The purpose of this paper is to socialize the results of a research, called "Characterization of teacher training in Humanities in Colombia", which aimed to explore trends and references regarding teacher training in the area of Humanities and Spanish Language in the country, after Decree 2450 of December 17, 2015 and Resolution 02041 of February 2016. For this purpose, the research was based on a descriptive analysis and on the parameters proposed by national policies in education and in the conceptual foundation on Humanities and Spanish Language. In this order of ideas, this paper presents a panorama of the educational policies that allow the programs to be structured and, later, it presents a descriptive analysis of the undergraduate programs in the Humanities area based on the concepts suggested by the regulations.
\end{abstract}

Keywords: Teacher training, Educational policies, Humanities, Spanish language.

\section{Introducción}

En el marco del campo educativo, la formación de profesores en humanidades y lengua castellana se considera relevante y pertinente, por una parte, porque permite atender las necesidades de alfabetización del país y la tarea de entender la comunicación 
como un mecanismo de consenso; y por la otra, porque ayuda a la comprensión de los constantes cambios en los valores, en las razones éticas y políticas del sujeto en la sociedad contemporánea.

Por ello, los procesos académicos desarrollados en las licenciaturas en esta área propenden por la formación de profesionales con capacidades pedagógicas, investigativas y comunicativas, que reflexionen e innoven sobre los procesos didácticos de su área de conocimiento, y además incentiven procesos comprometidos con el desarrollo cultural y académico de las comunidades.

En ese orden de ideas, el presente documento recoge parte de los resultados de un estudio descriptivo sobre las propuestas curriculares de las licenciaturas en humanidades y lengua castellana en Colombia, antes y después de la implementación de la Resolución 02041 de febrero de 2016, la cual convocó a un cambio de denominación y de estructuración de los créditos en dichos programas. Para ello, se han recogido las discusiones, las recomendaciones y los acuerdos de una serie de asesores y representantes de las diversas universidades con los programas del área mencionada a nivel nacional.

En esta medida, los aspectos aquí tratados permiten, en primera instancia, reflexionar sobre los cambios propuestos por el Ministerio de Educación en torno a las licenciaturas; en segundo lugar, atender los tránsitos que han generado las instituciones de educación superior en su fundamentación epistemológica, las propuestas didácticas y las prácticas pedagógicas en coherencia con los retos que surgen en la actualidad.

\section{Fundamentación conceptual de las políticas educativas}

En el sistema colombiano de formación de educadores se puede destacar la Ley 115 de 1994, la cual define que los programas de formación complementaria y de licenciatura deben estar acredita- 
dos de forma previa (cap. III, art. 113) según regulación y disposición a cargo del Consejo Nacional de Educación Superior (CESU), que dentro de sus funciones creó la Comisión Nacional Intersectorial de Aseguramiento de la Calidad de la Educación Superior (Conaces) y el Consejo Nacional de Acreditación (CNA), organismos encargados de la calidad de los programas de educación superior en el país.

Una de las labores de estas dos entidades se relaciona directamente con la emisión del registro calificado, con el cual se otorga el aval para el funcionamiento de un programa académico, siendo este el requisito inicial para ingresar al Sistema de Acreditación. En esa medida, los procesos de evaluación y acreditación de programas inician con la implementación del Acuerdo 06 del 14 de diciembre de 1995 del CESU. Estos procesos de acreditación se caracterizan por su rigurosidad y temporalidad; además, se debe destacar que "no es un instrumento de homogenización, ni jerarquización de las instituciones; por el contrario, busca promover el fortalecimiento de la evaluación académica y mejorar los niveles de calidad" (Perdomo y Buitrago, 2018, p. 11).

En los últimos años los procesos de acreditación han ido formalizándose y ha ido creciendo el número de universidades que se vinculan al proceso con el fin de participar en el mejoramiento continuo que se pretenden con los lineamientos de la acreditación; sin embargo, los programas que responden a las ciencias de la educación son los que muestran la proporción más baja en acudir a este mecanismo de validación de la calidad, tal como se evidencia en la evaluación realizada entre los años 1998-2008 (Garcés, 2008).

En consecuencia, el Ministerio de Educación Nacional divulgó la Resolución 02041 de febrero de 2016, "por la cual se establecen las características específicas de calidad de los programas de licenciatura para la obtención, renovación o modificación del registro calificado"; por tal razón, todos los programas de formación de profesores del país se vieron obligados a renovar sus propuestas curriculares. Esta reforma propuso delimitar la denominación de dichos programas y la estructuración de los currículos en rela- 
ción con cuatro componentes transversales: fundamentos generales, saberes específicos y disciplinares, pedagogía y ciencias de la educación y didácticas específicas; además de ello, esta resolución promueve la ampliación de los procesos de práctica e investigación.

Por consiguiente, a partir de 2016 las licenciaturas tuvieron un cambio significativo al implementar la nueva resolución, lo que generó un giro en la oferta de este tipo de programas a nivel nacional e inicialmente en los nombres de los programas, puesto que la norma delimita las denominaciones a diez en el caso del área de las licenciaturas en humanidades, lengua castellana e idiomas extranjeros.

En ese mismo sentido, los programas debieron asumir cambios en sus contenidos académicos; de acuerdo con el CNA (2013), los criterios curriculares son principios esenciales de la organización de las licenciaturas, ya que debido a estos las actividades académicas, formales y no formales, logran estructurarse y hacerse significativas en función de la propuesta de formación de los proyectos curriculares. De ahí que la flexibilidad, la interdisciplinariedad, la integralidad y la transdisciplinariedad se consoliden como los criterios curriculares en los que se estructura la formación integral promulgada en las políticas educativas.

La flexibilidad se entiende como la propiedad que tiene el currículo para promover la integralidad entre los saberes desde las exigencias de la disciplina a nivel nacional e internacional, garantizando la interacción de sus diversos componentes y propendiendo por una educación holística. En términos generales, las iniciativas desde las políticas educativas para promover la flexibilidad se centran en la inclusión del sistema de créditos, lo cual facilita la movilidad de los estudiantes al interior de su carrera, de su facultad o de su institución, e incluso la movilidad en otras instituciones.

De otro lado, la interdisciplinariedad se entiende como el criterio que contribuye a la interacción de diversas disciplinas para la solución de problemas ligados al programa o a problemas del 
entorno, para que el proceso educativo no se limite a los aspectos conceptuales y se enriquezca con los diversos campos del saber. La interdisciplinariedad es uno de los ejes que contribuye en la articulación de los proyectos de investigación, las prácticas sociales y profesionales, lo que es un mecanismo explícito de integración de unidades académicas en torno a problemas propios de la disciplina (CNA, 2013, p. 30).

En tercer lugar está la integralidad, que se define como la posibilidad que tiene el currículo para promover la interacción entre los saberes, garantizando la coherencia de sus diversos componentes. En términos generales, se expresa desde las relaciones establecidas por los contenidos curriculares con el perfil profesional, buscando la formación holística del estudiante. Por lo tanto, la formación integral se asume a partir de la concepción de un ser humano en permanente desarrollo y construcción, en relación con sus múltiples dimensiones: ética, moral, estética, social y política, con capacidades y potencialidades para incidir de manera crítica y dinámica en la transformación de sí mismo, del devenir histórico y de la sociedad.

Por último, en el marco de los criterios curriculares está la transdisciplinariedad, que se entiende como la forma de pensamiento relacional y como la interpretación del conocimiento desde la perspectiva de la vida humana y el compromiso social. Para ello, el currículo se abre a la necesidad de desarrollar un pensamiento complejo que vincule las disciplinas como posibilidad de humanidad superando la fragmentación entre la experiencia del estudiante y la búsqueda del conocimiento (Nicolescu, 2006, p. 23). En esta lógica, la transdisciplinariedad favorece la pluralidad de espacios académicos y extracurriculares que hagan posible enriquecer las dimensiones cognitiva, investigativa, cultural y espiritual del estudiante.

Por consiguiente, la flexibilidad, la interdisciplinariedad, la integralidad y la transdisciplinariedad se consolidan como elementos transversales y unificadores en el currículo. Así, se puede hablar de una perspectiva holística en la formación de educado- 
res que posibilita la pluralidad de saberes desde los programas académicos. Estos elementos transversales son los principios de los componentes que estructuran los currículos en educación, los cuales buscan desarrollar los procesos de formación a partir de los fundamentos generales, saberes específicos y disciplinares, pedagogía y ciencias de la educación y didácticas específicas.

Desde el componente de fundamentos generales se pretende el desarrollo de las habilidades comunicativas, el razonamiento matemático y cuantitativo, las competencias ciudadanas y el uso de las tecnologías de la innovación. A partir del componente de saberes específicos y disciplinares se busca consolidar, en el futuro docente, los fundamentos conceptuales del área en que se desempeñará. Adicionalmente, se convoca a desarrollar la capacidad de investigar, innovar y profundizar de forma autónoma en el conocimiento de dichos fundamentos. Con base en el componente de pedagogía y ciencias de la educación, se incita a llevar a la práctica los conocimientos pedagógicos y de las ciencias de la educación que permitan crear ambientes para la formación integral y el aprendizaje. Por último, en el componente de didácticas específicas se resalta la importancia de la articulación entre la pedagogía y la didáctica como fundamentos del quehacer del educador (Resolución 02041 de febrero de 2016).

En resumen, los principios curriculares y los componentes sugeridos desde las políticas nacionales de educación se convierten en el fundamento epistemológico para la estructuración del currículo. En esa medida, un currículo se puede entender como el conjunto de los principios epistemológicos, formativos, didácticos y evaluativos que erigen la misión, la visión y los objetivos del proceso de formación integral propuesto por los programas académicos. 


\section{Marco metodológico para la caracterización de las licenciaturas en humanidades}

Para el desarrollo de este proceso investigativo se utilizó un enfoque y diseño cualitativo, ya que este privilegia el análisis del objeto de estudio y sus particularidades. En palabras de Alejandra Reguera, la investigación cualitativa permite describir fenómenos que ocurren en un contexto social y cultural, sin la necesidad de llevarlos al plano de la experimentación (2008, p. 78). En consecuencia, para ejercicios de indagación como este, el enfoque cualitativo permite desarrollar procesos descriptivos e interpretativos que se caracterizan por que los investigadores, al plantear un problema, atienden sus realidades inmediatas, sin establecer un proceso estricto.

Así mismo, esta investigación se apoyó en los estudios descriptivos debido a la naturaleza social del objeto de estudio y a los procedimientos de análisis implementados. Estos estudios, relacionados con las ciencias humanas y sociales, proveen las herramientas apropiadas para lograr la interpretación del fenómeno estudiado. De la misma forma, ofrecen estrategias pertinentes para la observación, lo que posibilita a los investigadores identificar y analizar los hechos o datos relevantes de los documentos analizados. Siendo así, una metodología descriptiva permite aproximarse a los significados que subyacen en los proyectos curriculares que sustentan la formación de docentes en el área de las humanidades en el país.

Desde lo anterior, este estudio descriptivo pretende "familiarizarse con un fenómeno social y adquirir nuevos conocimientos sobre el mismo con el fin de formular un problema más preciso" (Reguera, 2008, p. 45); también busca realizar un análisis descriptivo desde la praxis investigativa. Para tal efecto, se consideran los elementos fundamentales expuestos por Hernández, Fernández y Baptista, quienes afirman que "los estudios descriptivos buscan especificar las propiedades, las características y los perfiles de 
personas, grupos, comunidades, procesos, objetos o cualquier otro fenómeno que se someta a un análisis" (2006, p. 102).

La revisión documental (de los proyectos curriculares) y la construcción de un marco referencial sobre la formación en humanidades se pueden entender como una práctica social, puesto que es un ejercicio en el que se pretende develar el estado actual de los procesos académicos en dicha formación. Por lo tanto, centrarse en un objeto de estudio con tales características requiere de seis etapas básicas para lograr su objetivo, las cuales parten del diseño de la investigación cualitativa y son coherentes con los estudios descriptivos propuestos por Baeza (2002).

La primera etapa es la identificación del problema de investigación, en la que se propone, explícita y sistemáticamente, construir conocimiento o crear un espacio de reflexión sobre la comprensión de los documentos que conforman la propuesta curricular de cada programa académico. La siguiente etapa es la presentación de una propuesta metodológica que parte del problema construido, es decir, depende de los objetivos específicos de la investigación. En la etapa a seguir se lleva a cabo el proceso de documentación, en el que se recoge la información pertinente en torno a los aspectos teórico-conceptuales. En cuarto lugar, se propone el diseño de un instrumento de recolección de datos, con base en una serie de categorías que se aplican para interpretar los proyectos curriculares. Posteriormente, se realiza el análisis, a partir de una lectura comparativa, de las propuestas académicas para llegar a una interpretación; y, finalmente, se socializan los hallazgos obtenidos a partir de los objetivos planteados. Así, el análisis de los documentos curriculares se consolida como un proceso investigativo enmarcado en el enfoque y el diseño cualitativo, que aborda un fenómeno social a partir de un estudio descriptivo.

De acuerdo con la estrategia metodológica de esta investigación, las técnicas de recolección y producción de información propuestas son las siguientes: revisión de las actuales políticas educativas nacionales sobre la formación de docentes en el área de humanidades, análisis de los currículos en el área de humanidades 
y lengua castellana (proyectos curriculares de las universidades nacionales), implementación de una encuesta, discusión e interpretación de resultados con pares colaborativos de las universidades nacionales y socialización de los resultados a la comunidad académica.

Como se mencionó previamente, en la implementación de dichas técnicas se recurrió a la encuesta, puesto que es "un instrumento que revela, a partir de un cierto número de individuos, las relaciones generales entre las características de un gran número de variables, mediante un procedimiento estímulo-respuesta homogéneo" (Gallardo y Moreno, 1999, p. 46). La encuesta de esta investigación fue ejecutada a través de correo electrónico y convocó a la participación de treinta y ocho universidades, y ofreció, además, un espacio abierto para comentar otros aspectos que se consideraron relevantes de los programas. Este instrumento se diseñó a partir de las siguientes variables (tabla 1 ).

Tabla 1. Variables del estudio descriptivo.

\begin{tabular}{|l|l|l|}
\hline Variable & Descripción & Hipótesis \\
\hline $\begin{array}{l}\text { Denominación del } \\
\text { programa }\end{array}$ & $\begin{array}{l}\text { El nombre actual del programa } \\
\text { de formación, el cual debe } \\
\text { responder a lo estipulado en la } \\
\text { Resolución. }\end{array}$ & $\begin{array}{l}\text { Los programas que optan por los } \\
\text { idiomas extranjeros pretenden crear un } \\
\text { marco más amplio de empleabilidad a } \\
\text { los egresados. Los programas centrados } \\
\text { en la lengua castellana buscan } \\
\text { consolidar procesos más específicos en } \\
\text { un área determinada. }\end{array}$ \\
\hline $\begin{array}{l}\text { Anterior denominación } \\
\text { del programa }\end{array}$ & $\begin{array}{l}\text { La denominación anterior a la } \\
\text { reforma contribuye a evidenciar } \\
\text { el cambio estructural del } \\
\text { programa. }\end{array}$ & $\begin{array}{l}\text { La comparación entre las } \\
\text { denominaciones actuales y anteriores } \\
\text { permite evidenciar el cambio } \\
\text { epistemológico de cada programa o la } \\
\text { consolidación de la propuesta anterior. }\end{array}$ \\
\hline Área de conocimiento & $\begin{array}{l}\text { El área del concomimiento } \\
\text { propuesta por el SNIES. }\end{array}$ & $\begin{array}{l}\text { Las licenciaturas se mantienen en el } \\
\text { campo de conocimiento de la educación } \\
\text { o pueden aproximarse al área de las } \\
\text { ciencias sociales o al campo de las } \\
\text { humanidades. Esto contribuye a analizar } \\
\text { el área específica del programa. }\end{array}$ \\
\hline Número de créditos: & $\begin{array}{l}\text { Duración del programa en } \\
\text { créditos académicos }\end{array}$ & $\begin{array}{l}\text { Las licenciaturas con metodología } \\
\text { presencial proponen un mayor } \\
\text { acercamiento al estudiante. Los } \\
\text { programas virtuales y a distancia } \\
\text { convocan a mayor autonomía por parte } \\
\text { del estudiante. }\end{array}$ \\
\hline & $\begin{array}{l}\text { La cantidad de créditos permite } \\
\text { evidenciar la flexibilidad y la } \\
\text { transversalidad del programa. }\end{array}$ \\
\hline & $\begin{array}{l}\text { Presencial/virtual/a distancia } \\
\text { Metodología }\end{array}$ & \\
\hline
\end{tabular}




\begin{tabular}{|l|l|l|}
\hline Variable & Descripción & Hipótesis \\
\hline Duración & $\begin{array}{l}\text { La extensión del programa } \\
\text { en semestres, trimestres o } \\
\text { cuatrimestres. }\end{array}$ & $\begin{array}{l}\text { Los programas con mayor cantidad de } \\
\text { créditos buscan especializarse en las } \\
\text { áreas de conocimiento. Los programas } \\
\text { con menor cantidad de créditos } \\
\text { proponen mayor flexibilidad y autonomía } \\
\text { para los estudiantes. }\end{array}$ \\
\hline $\begin{array}{l}\text { Principios que orientan } \\
\text { el programa }\end{array}$ & $\begin{array}{l}\text { Fundamentos epistemológicos } \\
\text { del programa }\end{array}$ & $\begin{array}{l}\text { Los principios que fundan el programa } \\
\text { pueden centrarse en la flexibilidad, la } \\
\text { interdisciplinariedad, la integralidad y la } \\
\text { transdisciplinariedad. }\end{array}$ \\
\hline
\end{tabular}

Fuente: elaboración propia.

\section{Análisis descriptivo de las propuestas curriculares en humanidades y lengua castellana}

En el desarrollo de esta investigación se realizó un análisis de las propuestas curriculares en torno al área de interés; para ello se revisaron programas de Humanidades, Lengua Castellana, Literatura, y Lenguas en función de identificar lo particular y universal de cada uno de los programas. Como antecedente de este propósito se retomó un estudio realizado en varias universidades del país (Rincón, 2004)³, el cual permitió identificar algunas tendencias en la formación de docentes en esta área a nivel iberoamericano en ese momento.

Este estudio tuvo como propósito realizar un análisis sobre la formación de docentes en lengua castellana en el 2004, previo a la implementación de las Pruebas de Calidad de la Educación Superior (Ecaes). Así mismo, en este trabajo se presentó un marco referencial en torno a la formación de profesionales en el área de la lengua castellana y afines a nivel nacional e internacional. En esa medida, se efectuó "una revisión de la discusión pedagógica, didáctica y de las disciplinas del énfasis, sobre la formación de docentes para las licenciaturas [...] a nivel internacional" (Rincón, 2004, p. 9).

\footnotetext{
3. El propósito de este estudio es presentar un análisis sobre la formación de docentes en lengua castellana que fue tomado como referencia conceptual para la elaboración del Examen de Calidad de la Educación Superior (Ecaes). También en este trabajo se realiza un estudio descriptivo de las características de los programas acreditados hasta el 2004 en el país y los perfiles de egresados que ellos plantean. En este estudio comparado se definen los aspectos comunes y centrales de los programas ofrecidos por las diferentes facultades.
} 
En esta revisión de los programas académicos latinoamericanos se evidenciaron algunas tendencias en relación con la denominación y el desarrollo de los contenidos de las asignaturas. La lectura transversal de dichos programas develó que en la mayoría de las licenciaturas eran escasas las asignaturas referidas a la pedagogía y la didáctica. Al respecto, se mencionaba que era "más frecuente encontrar asignaturas referidas a materiales didácticos que a la didáctica como un campo de saber" (Rincón, 2004, p. 17). Por consiguiente, en Latinoamérica en ese entonces existía una tendencia a privilegiar el trabajo en torno a la teoría de la lengua y no a las didácticas específicas.

Con este mismo objetivo, en una revisión más reciente de la formación de docentes en lengua castellana en Colombia, en el año 2015 se llevó a cabo un estudio que evidenciaba que las tendencias develadas anteriormente habían variado muy poco en la última década; además de ello, se observaba que en el contexto nacional de la formación en lengua castellana y áreas afines había una tendencia a denominar sus programas enfatizando en las humanidades (Perdomo, Buitrago y Báez, 2017).

En el desarrollo de este estudio se descartaron los programas académicos que se centraban directamente en los estudios literarios y las ciencias del lenguaje, puesto que su misión no se centraba en el ámbito educativo. Así, se seleccionaron cuarenta y dos licenciaturas activas en el 2015 en el país (Perdomo, López y Mahecha, 2016, p. 141).

En resumen, de acuerdo con los datos del SNIES ${ }^{4}$ del año 2015 se podían encontrar las siguientes denominaciones de licenciaturas en humanidades, lengua castellana y áreas afines (tabla 2).

\footnotetext{
4. El Sistema Nacional de Información de la Educación Superior (SNIES) es un sistema de información que ha sido creado para responder a las necesidades de información de la educación superior en Colombia. En este sistema se recopila y organiza la información relevante sobre la educación superior que permite hacer planeación, monitoreo, evaluación, asesoría, inspección y vigilancia del sector.
} 
Tabla 2. Denominaciones de los programas en humanidades o áreas afines en 2015.

\begin{tabular}{|c|c|}
\hline Denominación & $\begin{array}{l}\text { Cantidad de } \\
\text { programas }\end{array}$ \\
\hline $\begin{array}{l}\text { Licenciaturas en Educación Básica con Énfasis en Humanidades y Lengua } \\
\text { Castellana }\end{array}$ & 6 \\
\hline $\begin{array}{l}\text { Licenciaturas en Educación Básica con Énfasis en Humanidades y Lengua } \\
\text { Castellana e Inglés }\end{array}$ & 4 \\
\hline Licenciaturas en Educación Básica con Énfasis en Lengua Castellana & 2 \\
\hline $\begin{array}{l}\text { Licenciatura en Educación Básica con Énfasis en Humanidades, Lengua } \\
\text { Castellana y Lenguas }\end{array}$ & 1 \\
\hline $\begin{array}{l}\text { Licenciatura en Educación Básica con Énfasis en Matemáticas, Humanidades y } \\
\text { Lengua Castellana }\end{array}$ & 1 \\
\hline $\begin{array}{l}\text { Licenciatura en Educación Básica con Énfasis en Humanidades: Español e } \\
\text { Inglés }\end{array}$ & 1 \\
\hline Licenciatura en Educación Básica con Énfasis en Humanidades y Lenguas & 1 \\
\hline Licenciatura Educación Básica con Énfasis en Humanidades e Idiomas & 1 \\
\hline Licenciatura en Educación Básica con Énfasis en Humanidades & 1 \\
\hline Licenciatura en Literatura y Lengua Castellana & 1 \\
\hline Licenciatura en Español e Inglés & 1 \\
\hline Licenciatura en Lengua Castellana & 5 \\
\hline Licenciatura en Lengua Castellana y Literatura & 2 \\
\hline Licenciatura en Lengua Castellana e Inglés & 3 \\
\hline Licenciatura en Español y Literatura & 4 \\
\hline Licenciatura en Literatura & 1 \\
\hline Licenciatura en Lingüística y Literatura & 2 \\
\hline Licenciatura en Lengua Castellana y Comunicación & 1 \\
\hline Licenciatura en Filosofía y Humanidades & 1 \\
\hline
\end{tabular}




\begin{tabular}{|l|l|}
\hline Denominación & $\begin{array}{l}\text { Cantidad de } \\
\text { programas }\end{array}$ \\
\hline Licenciatura en Lenguas Modernas & 1 \\
\hline Licenciatura en Filosofía y Lengua Castellana & 1 \\
\hline Licenciatura en Lengua Castellana, Inglés y Francés & 1 \\
\hline
\end{tabular}

Fuente: Perdomo, López y Mahecha (2016, p. 141).

En consecuencia, en Colombia se registraban dieciocho programas que ofrecían la licenciatura en Educación Básica con algún tipo de énfasis relacionado con las humanidades o la lengua castellana (figura 1); entre ellas, siete vinculaban las humanidades o la lengua castellana con inglés u otro idioma extranjero; además, se encontraba una licenciatura que mencionaba en su denominación las matemáticas. También existían veinticuatro que no consideraban en su titulación el campo de desempeño de la educación básica, entre las cuales una relacionaba la filosofía con las humanidades y otra con la comunicación.

Figura 1. Porcentaje de denominaciones de licenciatura en humanidades y áreas afines en 2015.

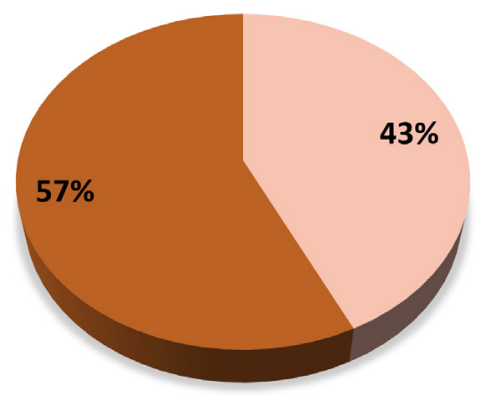

Con énfasis en Educación Básica: Humanidades, Lengua

Castellana, Inglés,Matemáticas

- Sin énfasis en Educación Básica: Lenguas, Filosofía, Comunicación

Fuente: creación propia. 
Adicionalmente, se evidenciaba que un gran número de programas optaban por obviar educación básica para denominar el programa en relación directa con el área disciplinar (24 programas de un total de 42). También era clara la diversidad en relación con el número de créditos ofertados, entre 131 y 180 créditos.

El estudio mencionado, las tendencias allí enmarcadas y las reflexiones sobre ellas contribuyeron al objetivo de esta investigación, el cual consiste en realizar una aproximación al estado de los programas académicos en humanidades y lengua castellana en el contexto nacional, en relación con su denominación y propuesta de créditos, después de la implementación y entrada en vigencia del Decreto 2450 del 17 de diciembre de 2015 y de la Resolución 02041 del 3 de febrero de 2016.

Los criterios de dicha normatividad fueron una base para analizar nuevamente las mismas licenciaturas desde su titulación. En esa medida, de acuerdo con el SNIES y en comparación con el estudio del 2015, se puede evidenciar que en el año 2020 del primer grupo (de dieciocho programas), el enfocado en la educación básica, ocho definen en su denominación el trabajo centrado en las humanidades, la lengua castellana o afines (tabla 3 ).

Tabla 3. Comparación de las denominaciones de Licenciaturas en Educación Básica con Énfasis en Lengua Castellana y Literatura (2015-2020)

\begin{tabular}{|l|l|l|l|l|}
\hline Universidad & $\begin{array}{l}\text { Denominación anterior a } \\
\text { la Resolución 02041 }\end{array}$ & Créditos & $\begin{array}{l}\text { Denominación } \\
\text { posterior a la } \\
\text { Resolución 02041 }\end{array}$ & Créditos \\
\hline $\begin{array}{l}\text { Corporación } \\
\text { Universitaria del } \\
\text { Caribe }\end{array}$ & $\begin{array}{l}\text { Licenciatura en Educación } \\
\text { Básica con Énfasis en } \\
\text { Humanidades }\end{array}$ & 153 & $\begin{array}{l}\text { Licenciatura en } \\
\text { Lingüística y Literatura }\end{array}$ & 156 \\
\hline $\begin{array}{l}\text { Universidad de } \\
\text { Córdoba }\end{array}$ & $\begin{array}{l}\text { Licenciatura en Educación } \\
\text { Básica con Énfasis en } \\
\text { Humanidades - Lengua } \\
\text { Castellana }\end{array}$ & 154 & $\begin{array}{l}\text { Licenciatura en } \\
\text { Literatura y Lengua } \\
\text { Castellana }\end{array}$ & 163 \\
\hline $\begin{array}{l}\text { Universidad Distrital } \\
- \text { Francisco José de } \\
\text { Caldas }\end{array}$ & $\begin{array}{l}\text { Licenciatura en Educación } \\
\text { básica con Énfasis en } \\
\text { Humanidades y Lengua } \\
\text { Castellana }\end{array}$ & 179 & $\begin{array}{l}\text { Licenciatura en } \\
\text { Humanidades y Lengua } \\
\text { Castellana }\end{array}$ & 154 \\
\hline $\begin{array}{l}\text { Universidad del } \\
\text { Magdalena }\end{array}$ & $\begin{array}{l}\text { Licenciatura en Educación } \\
\text { Básica con Énfasis en } \\
\text { Humanidades y Lengua } \\
\text { Castellana }\end{array}$ & 145 & $\begin{array}{l}\text { Licenciatura en } \\
\text { Literatura y Lengua } \\
\text { Castellana }\end{array}$ & 158 \\
\hline
\end{tabular}




\begin{tabular}{|c|c|c|c|c|}
\hline Universidad & $\begin{array}{l}\text { Denominación anterior a } \\
\text { la Resolución } 02041\end{array}$ & Créditos & $\begin{array}{l}\text { Denominación } \\
\text { posterior a la } \\
\text { Resolución } 02041\end{array}$ & Créditos \\
\hline $\begin{array}{l}\text { Universidad } \\
\text { Pedagógica y } \\
\text { Tecnológica de } \\
\text { Colombia }\end{array}$ & $\begin{array}{l}\text { Licenciatura en Educación } \\
\text { Básica con Énfasis en } \\
\text { Matemáticas y Español }\end{array}$ & 152 & $\begin{array}{l}\text { Licenciatura en } \\
\text { Literatura y Lengua } \\
\text { Castellana }\end{array}$ & 159 \\
\hline $\begin{array}{l}\text { Universidad de } \\
\text { Antioquia }\end{array}$ & $\begin{array}{l}\text { Licenciatura en Educación } \\
\text { Básica con Énfasis en } \\
\text { Humanidades y Lengua } \\
\text { Castellana }\end{array}$ & 177 & $\begin{array}{l}\text { Licenciatura en } \\
\text { Literatura y Lengua } \\
\text { Castellana }\end{array}$ & 179 \\
\hline $\begin{array}{l}\text { Universidad } \\
\text { Tecnológica de } \\
\text { Antioquia }\end{array}$ & $\begin{array}{l}\text { Licenciatura en Educación } \\
\text { Básica con Énfasis en } \\
\text { Humanidades y Lengua } \\
\text { Castellana }\end{array}$ & 168 & $\begin{array}{l}\text { Licenciatura en } \\
\text { Literatura y Lengua } \\
\text { Castellana }\end{array}$ & 160 \\
\hline $\begin{array}{l}\text { Corporación } \\
\text { Universitaria Minuto } \\
\text { de Dios }\end{array}$ & $\begin{array}{l}\text { Licenciatura en Educación } \\
\text { Básica con Énfasis en } \\
\text { Humanidades y Lengua } \\
\text { Castellana }\end{array}$ & 144 & $\begin{array}{l}\text { Licenciatura en } \\
\text { Humanidades y Lengua } \\
\text { Castellana }\end{array}$ & 155 \\
\hline
\end{tabular}

Fuente: creación propia.

Como se presenta en la tabla 3, las licenciaturas de este grupo no variaron considerablemente su denominación; continúa su interés por desarrollar los procesos desde la lengua castellana. Sin embargo, cabe destacar que, como lo sugiere la resolución, todos los programas eliminaron de sus títulos el énfasis en educación básica. Esto se puede interpretar como un mecanismo para ampliar las posibilidades de desempeño laboral de sus egresados y para asegurar su inserción en el sistema productivo como lo contempla el Decreto 2450.

La denominación de dicho énfasis puede limitar el trabajo de los egresados de las licenciaturas a dos de los ciclos de la educación: la educación básica primaria de cinco grados y la educación básica secundaria de cuatro grados, sin permitir un ejercicio laboral en la educación media. En esta medida, se podría asegurar también que los programas mencionados cuentan con un gran índice de flexibilidad, representada en la amplitud de sus espacios y temáticas de trabajo, que puede visualizarse también en la suma y distribución de sus créditos académicos; en cuanto a la integralidad, cabe destacar que solo dos programas se decidieron por las humanidades, ambos de Bogotá, señalando en su título una apuesta por la formación con un compromiso ético, político, social y humano. Final- 
mente, es importante mencionar que ninguno de estos programas optó por la filología o la pura literatura apostándole a una formación interdisciplinar con amplitud en fundamentos conceptuales y disciplinares.

De ese primer grupo de dieciocho programas (tomado del estudio de 2015), las diez licenciaturas restantes se pueden dividir en dos, el primer subgrupo lo conforman siete programas que se encaminan por las lenguas o los idiomas, sin restarle importancia al español (tabla 4).

Tabla 4. Comparación de las denominaciones de Licenciaturas en Educación Básica con Énfasis en Lenguas Extranjeras e Idiomas (2015-2020)

\begin{tabular}{|c|c|c|c|c|}
\hline Universidad & $\begin{array}{l}\text { Denominación anterior a } \\
\text { la Resolución } 02041\end{array}$ & Créditos & $\begin{array}{l}\text { Denominación } \\
\text { posterior a la } \\
\text { Resolución } 02041 \\
\end{array}$ & Créditos \\
\hline Universidad de Nariño & $\begin{array}{l}\text { Licenciatura en Educación } \\
\text { Básica con Énfasis en } \\
\text { Humanidades, Lengua } \\
\text { Castellana e Inglés }\end{array}$ & 149 & $\begin{array}{l}\text { Licenciatura en Español } \\
\text { e Inglés }\end{array}$ & 152 \\
\hline $\begin{array}{l}\text { Corporación } \\
\text { Universitaria } \\
\text { Adventista (UNAC) }\end{array}$ & $\begin{array}{l}\text { Licenciatura en Educación } \\
\text { Básica con Énfasis en } \\
\text { Humanidades: Español e } \\
\text { Inglés }\end{array}$ & 144 & $\begin{array}{l}\text { Licenciatura en Español } \\
\text { e Inglés }\end{array}$ & 164 \\
\hline Universidad INCCA & $\begin{array}{l}\text { Licenciatura en Educación } \\
\text { Básica con Énfasis en } \\
\text { Humanidades y Lengua } \\
\text { Castellana e Inglés }\end{array}$ & 165 & $\begin{array}{l}\text { Licenciatura en } \\
\text { Humanidades y Lengua } \\
\text { Castellana e Inglés }\end{array}$ & 165 \\
\hline $\begin{array}{l}\text { Universidad } \\
\text { Pedagógica Nacional }\end{array}$ & $\begin{array}{l}\text { Licenciatura en Educación } \\
\text { Básica con Énfasis en } \\
\text { Humanidades y Lengua } \\
\text { Castellana e Inglés }\end{array}$ & 160 & $\begin{array}{l}\text { Licenciatura en Español } \\
\text { e Inglés }\end{array}$ & 160 \\
\hline $\begin{array}{l}\text { Universidad } \\
\text { Pedagógica Nacional }\end{array}$ & $\begin{array}{l}\text { Licenciatura en Educación } \\
\text { Básica con Énfasis en } \\
\text { Humanidades, Lengua } \\
\text { Castellana y Lenguas }\end{array}$ & 160 & $\begin{array}{l}\text { Licenciatura en Español } \\
\text { y Lenguas Extranjeras } \\
\text { con Énfasis en Inglés y } \\
\text { Francés }\end{array}$ & 160 \\
\hline $\begin{array}{l}\text { Universidad Libre } \\
\text { (Bogotá) }\end{array}$ & $\begin{array}{l}\text { Licenciatura en Educación } \\
\text { Básica con Énfasis en } \\
\text { Humanidades e Idiomas }\end{array}$ & 160 & $\begin{array}{l}\text { Licenciatura en Español } \\
\text { y Lenguas Extranjeras }\end{array}$ & 165 \\
\hline $\begin{array}{l}\text { Universidad Libre } \\
\text { (Socorro) }\end{array}$ & $\begin{array}{l}\text { Licenciatura en Educación } \\
\text { Básica con Énfasis en } \\
\text { Humanidades y Lenguas }\end{array}$ & 160 & $\begin{array}{l}\text { Licenciatura en Español } \\
\text { e Inglés }\end{array}$ & 165 \\
\hline
\end{tabular}

Fuente: creación propia. 
En general, la variación de créditos entre los programas anteriores y posteriores a la resolución es mínima, de entre 3 y 5 créditos, salvo el caso de una licenciatura que aumentó en 20 créditos su plan de estudios. En las denominaciones de los programas se puede observar cómo estos han especificado la lengua extranjera en la que formarán, teniendo en cuenta que según la normatividad se deberá evidenciar que los estudiantes logran un nivel superior en el manejo de la lengua que determina su título, puesto que en el caso de inglés los estudiantes deben mostrar un dominio en el nivel $\mathrm{C} 1$ de acuerdo con los estándares del Marco Común Europeo de Referencia (MCER), que serán verificados con los resultados de la prueba Saber Pro, con exámenes estandarizados o referidos en la Lista Actualizada de Exámenes que publica el Ministerio de Educación Nacional.

De esta manera, las instituciones de educación superior que ofertan una lengua distinta a la materna deben prever que sus egresados deberán acreditar un dominio superior, hecho que implica un fortalecimiento en sus currículos, además de adecuaciones y mejoras de las estrategias para el desarrollo de competencias en un segundo idioma. Es importante mencionar que estas licenciaturas también amplían las oportunidades laborales a las que podrán acceder sus egresados, teniendo en cuenta que el dominio de una segunda lengua se ha establecido como una forma oportuna de acceso al sector productivo, según las bases del Plan Nacional de Desarrollo 2014-2018.

En el segundo subgrupo se pueden encontrar las tres licenciaturas faltantes que no se siguieron ofertando (tabla 5). 
Tabla 5. Licenciaturas en Educación Básica con Énfasis en Lengua Castellana y afines que se dejaron de ofertar a partir de la Resolución 02041.

\begin{tabular}{|l|l|l|}
\hline Universidad & Denominación anterior a la Resolución 02041 & $\begin{array}{l}\text { Estado } \\
\text { actual } \\
\mathbf{( 2 0 2 0 )}\end{array}$ \\
\hline $\begin{array}{l}\text { Universidad Industrial } \\
\text { de Santander (UIS) }\end{array}$ & $\begin{array}{l}\text { Licenciatura en Educación Básica con Énfasis en Lengua } \\
\text { Castellana }\end{array}$ & No se oferta \\
\hline $\begin{array}{l}\text { Fundación } \\
\text { Universitaria } \\
\text { Monserrate }\end{array}$ & $\begin{array}{l}\text { Licenciatura en Educación Básica con Énfasis en Lengua } \\
\text { Castellana }\end{array}$ & No se oferta \\
\hline $\begin{array}{l}\text { Universidad de } \\
\text { Cundinamarca }\end{array}$ & $\begin{array}{l}\text { Licenciatura en Educación Básica con Énfasis en } \\
\text { Humanidades y Lengua Castellana e Inglés }\end{array}$ & No se oferta \\
\hline
\end{tabular}

Fuente: creación propia.

En el caso de la Universidad Industrial de Santander (UIS), esta institución ofertaba en 2015 dos programas relacionados con la lengua castellana y la literatura, respectivamente; en la actualidad oferta solo un programa con una denominación que elimina la educación básica y el énfasis en lengua castellana, optando por una titulación con mayor flexibilidad de contenidos curriculares y especificidad en la formación en literatura, que tiene como nombre Licenciatura en Literatura y Lengua Castellana, con 178 créditos. Por su parte, la Fundación Universitaria Monserrate, después del cambio de normativa, oferta una Licenciatura en Educación Bilingüe y una Licenciatura en Educación Básica Primaria, eliminando cualquier programa que contenga en su titulación la lengua casteIlana; este cambio se pudo dar si se tiene en cuenta que antes de la resolución la licenciatura ofertada tenía un componente curricular con gran cantidad de espacios dirigidos a la enseñanza, a la didáctica y a la práctica en la educación primaria, y si se reconoce, además, que la universidad ya contaba con un programa de formación en inglés con las mismas características. Finalmente, la Universidad de Cundinamarca no continúa ofertando la Licenciatura en Educación Básica con Énfasis en Humanidades y Lengua Castellana e Inglés ni ningún programa afín.

De otro lado, el grupo de veinticuatro programas que en 2015 descartaba la educación básica en su denominación también se divide en tres; el primero de ellos (dieciséis programas) se centran en la lengua castellana y la literatura (tabla 6). 
Tabla 6. Comparación de las denominaciones de Licenciaturas en Lengua Castellana (2015-2020).

\begin{tabular}{|c|c|c|c|c|}
\hline Universidad & $\begin{array}{l}\text { Denominación anterior a } \\
\text { la Resolución } 02041\end{array}$ & Créditos & $\begin{array}{l}\text { Denominación } \\
\text { posterior a la } \\
\text { Resolución } 02041\end{array}$ & Créditos \\
\hline Universidad de Nariño & $\begin{array}{l}\text { Licenciatura en Lengua } \\
\text { Castellana y Literatura }\end{array}$ & 156 & $\begin{array}{l}\text { Licenciatura en Lengua } \\
\text { Castellana y Literatura }\end{array}$ & 156 \\
\hline $\begin{array}{l}\text { Universidad Industrial } \\
\text { de Santander (UIS) }\end{array}$ & $\begin{array}{l}\text { Licenciatura en Español y } \\
\text { Literatura }\end{array}$ & 170 & $\begin{array}{l}\text { Licenciatura en } \\
\text { Literatura y Lengua } \\
\text { Castellana }\end{array}$ & 178 \\
\hline $\begin{array}{l}\text { Universidad de la } \\
\text { Amazonia }\end{array}$ & $\begin{array}{l}\text { Licenciatura en Lengua } \\
\text { Castellana y Literatura }\end{array}$ & 131 & $\begin{array}{l}\text { Licenciatura en } \\
\text { Literatura y Lengua } \\
\text { Castellana }\end{array}$ & 131 \\
\hline $\begin{array}{l}\text { Universidad de } \\
\text { Pamplona }\end{array}$ & $\begin{array}{l}\text { Licenciatura en Lengua } \\
\text { Castellana y Comunicación }\end{array}$ & 162 & $\begin{array}{l}\text { Licenciatura en } \\
\text { Humanidades y Lengua } \\
\text { Castellana }\end{array}$ & 164 \\
\hline $\begin{array}{l}\text { Universidad de San } \\
\text { Buenaventura (Cali) }\end{array}$ & $\begin{array}{l}\text { Licenciatura en Lengua } \\
\text { Castellana }\end{array}$ & 153 & $\begin{array}{l}\text { Licenciatura en } \\
\text { Literatura y Lengua } \\
\text { Castellana }\end{array}$ & 144 \\
\hline $\begin{array}{l}\text { Universidad Popular } \\
\text { del Cesar }\end{array}$ & $\begin{array}{l}\text { Licenciatura en Lengua } \\
\text { Castellana e Inglés }\end{array}$ & 170 & $\begin{array}{l}\text { Licenciatura en } \\
\text { Literatura y Lengua } \\
\text { Castellana }\end{array}$ & 160 \\
\hline Universidad del Valle & Licenciatura en Literatura & 171 & $\begin{array}{l}\text { Licenciatura en } \\
\text { Literatura }\end{array}$ & 171 \\
\hline $\begin{array}{l}\text { Universidad del } \\
\text { Quindío }\end{array}$ & $\begin{array}{l}\text { Licenciatura en Español y } \\
\text { Literatura }\end{array}$ & 159 & $\begin{array}{l}\text { Licenciatura en } \\
\text { Literatura y Lengua } \\
\text { Castellana }\end{array}$ & 160 \\
\hline $\begin{array}{l}\text { Universidad } \\
\text { Tecnológica de } \\
\text { Pereira }\end{array}$ & $\begin{array}{l}\text { Licenciatura en Español y } \\
\text { Literatura }\end{array}$ & 152 & $\begin{array}{l}\text { Licenciatura en } \\
\text { Literatura y Lengua } \\
\text { Castellana }\end{array}$ & 159 \\
\hline $\begin{array}{l}\text { Universidad del } \\
\text { Atlántico }\end{array}$ & $\begin{array}{l}\text { Licenciatura en Español y } \\
\text { Literatura }\end{array}$ & 140 & $\begin{array}{l}\text { Licenciatura en } \\
\text { Humanidades y Lengua } \\
\text { Castellana }\end{array}$ & 178 \\
\hline $\begin{array}{l}\text { Universidad del } \\
\text { Cauca }\end{array}$ & $\begin{array}{l}\text { Licenciatura en Español e } \\
\text { Inglés }\end{array}$ & 173 & $\begin{array}{l}\text { Licenciatura en } \\
\text { Literatura y Lengua } \\
\text { Castellana }\end{array}$ & 168 \\
\hline
\end{tabular}




\begin{tabular}{|l|l|l|l|l|}
\hline Universidad & $\begin{array}{l}\text { Denominación anterior a } \\
\text { la Resolución 02041 }\end{array}$ & Créditos & $\begin{array}{l}\text { Denominación } \\
\text { posterior a la } \\
\text { Resolución 02041 }\end{array}$ & Créditos \\
\hline $\begin{array}{l}\text { Universidad del } \\
\text { Tolima }\end{array}$ & $\begin{array}{l}\text { Licenciatura en Lengua } \\
\text { Castellana }\end{array}$ & 170 & $\begin{array}{l}\text { Licenciatura en } \\
\text { Literatura y Lengua } \\
\text { Castellana }\end{array}$ & 176 \\
\hline $\begin{array}{l}\text { Universidad La Gran } \\
\text { Colombia }\end{array}$ & $\begin{array}{l}\text { Licenciatura en Lingüística } \\
\text { Literatura }\end{array}$ & 155 & $\begin{array}{l}\text { Licenciatura en } \\
\text { Humanidades y Lengua } \\
\text { Castellana }\end{array}$ & 155 \\
\hline $\begin{array}{l}\text { Universidad } \\
\text { Surcolombiana }\end{array}$ & $\begin{array}{l}\text { Licenciatura en Lengua } \\
\text { Castellana }\end{array}$ & 155 & $\begin{array}{l}\text { Licenciatura en } \\
\text { Literatura y Lengua } \\
\text { Castellana }\end{array}$ & 165 \\
\hline $\begin{array}{l}\text { Universidad de la } \\
\text { Salle }\end{array}$ & $\begin{array}{l}\text { Licenciatura en Lengua } \\
\text { Castellana, Inglés y Francés }\end{array}$ & 180 & $\begin{array}{l}\text { Licenciatura en } \\
\text { Literatura y Lengua } \\
\text { Castellana }\end{array}$ & 155 \\
\hline $\begin{array}{l}\text { Universidad } \\
\text { Chocó D. L. C. }\end{array}$ & $\begin{array}{l}\text { Licenciatura en Lingüística } \\
\text { Literatura }\end{array}$ & 147 & $\begin{array}{l}\text { Licenciatura en } \\
\text { Castellana }\end{array}$ & 161 \\
\hline
\end{tabular}

Fuente: creación propia.

Los programas que se centraban en lengua castellana y lengua extranjera optaron en su nueva denominación solo por la primera, y además disminuyeron el número de sus créditos atendiendo a la flexibilización en el currículo que supone trabajar con un solo idioma y no dos. Por otra parte, pocas licenciaturas optaron por incluir en sus nombres las humanidades; solo dos lo hicieron manifestando un carácter transversal de sus planes de estudio. La mayoría de los programas optó por literatura y lengua castellana, lo que permite determinar una formación curricular fuerte en estos conceptos disciplinares. Sin embargo, cabe destacar que esto no implica que los programas dejen a un lado otros elementos como la integralidad.

El segundo grupo corresponde a dos programas que ofertaban la lengua castellana con una lengua extranjera o lenguas modernas. Una de estas licenciaturas mantuvo su número de créditos, mientras que la otra los aumentó en 6; pese a esto, ambos programas mantienen su trabajo transversal ofertando un currículo para la formación en ambos idiomas (tabla 7). 
Tabla 7. Comparación de denominaciones de licenciaturas en lenguas extranjeras o idiomas (2015-2020).

\begin{tabular}{|l|l|l|l|l|}
\hline Universidad & $\begin{array}{l}\text { Denominación anterior a } \\
\text { la Resolución 02041 }\end{array}$ & Créditos & $\begin{array}{l}\text { Denominación } \\
\text { posterior a la } \\
\text { Resolución 02041 }\end{array}$ & Créditos \\
\hline $\begin{array}{l}\text { Universidad Antonio } \\
\text { Nariño }\end{array}$ & $\begin{array}{l}\text { Licenciatura en Lengua } \\
\text { Castellana e Inglés }\end{array}$ & 144 & $\begin{array}{l}\text { Licenciatura en Español } \\
\text { e Inglés }\end{array}$ & 150 \\
\hline $\begin{array}{l}\text { Pontificia Universidad } \\
\text { Javeriana }\end{array}$ & $\begin{array}{l}\text { Licenciatura en Lenguas } \\
\text { Modernas }\end{array}$ & 180 & $\begin{array}{l}\text { Modernas con Énfasis } \\
\text { en Inglés y Francés }\end{array}$ & 180 \\
\hline
\end{tabular}

Fuente: creación propia.

El último subgrupo lo conforman las licenciaturas que no se ofertan en el 2020, son seis programas que debido a los cambios propuestos por la Resolución 02041 decidieron no continuar:

Tabla 8. Programas en lengua castellana y letras que no se ofertan en el 2020.

\begin{tabular}{|l|l|l|l|}
\hline Universidad & Denominación anterior a la Resolución 02041 & Créditos & $\begin{array}{l}\text { Estado } \\
\text { actual }\end{array}$ \\
\hline $\begin{array}{l}\text { Corporación } \\
\text { Universitaria del } \\
\text { Caribe (Presencial) }\end{array}$ & Licenciatura en Literatura y Lengua Castellana & 155 & $\begin{array}{l}\text { No se } \\
\text { oferta }\end{array}$ \\
\hline $\begin{array}{l}\text { Universidad Santo } \\
\text { Tomas }\end{array}$ & Licenciatura en Filosofía y Lengua Castellana & 158 & $\begin{array}{l}\text { No se } \\
\text { oferta }\end{array}$ \\
\hline $\begin{array}{l}\text { Universidad Católica } \\
\text { del Norte }\end{array}$ & Licenciatura en Lengua Castellana & 136 & $\begin{array}{l}\text { No se } \\
\text { oferta }\end{array}$ \\
\hline $\begin{array}{l}\text { Fundación } \\
\text { Universitaria Luis } \\
\text { Amigó }\end{array}$ & Licenciatura en Lengua Castellana (Distancia) & 144 & $\begin{array}{l}\text { No se } \\
\text { oferta }\end{array}$ \\
\hline $\begin{array}{l}\text { Universidad } \\
\text { Cooperativa } \\
\text { de Colombia } \\
\text { (Bucaramanga) }\end{array}$ & Licenciatura en Lengua Castellana e Inglés & 140 & $\begin{array}{l}\text { No se } \\
\text { oferta }\end{array}$ \\
\hline $\begin{array}{l}\text { Universidad Sergio } \\
\text { Arboleda }\end{array}$ & Licenciatura en Filosofía y Letras & 150 & $\begin{array}{l}\text { No se } \\
\text { oferta }\end{array}$ \\
\hline
\end{tabular}

Fuente: creación propia.

Por otro lado, un grupo adicional lo compone la Licenciatura en Literatura y Lengua Castellana de la Pontificia Universidad Javeriana. Este es un programa nuevo que fue creado después de la normatividad de 2015 y 2016. En la actualidad, se oferta desde el 18 de diciembre de 2019, y aunque su denominación no es diferente 
a la de otras licenciaturas existentes, dentro de sus 144 créditos se puede observar una atención especial a los contenidos curriculares y competencias formativas exigidas por la ley, además de un compromiso por las nuevas demandas pedagógicas y didácticas.

Figura 2. Comparación de las licenciaturas por cantidad de créditos en 2015 y 2020.

Comparativa de créditos licenciaturas 2015-2020

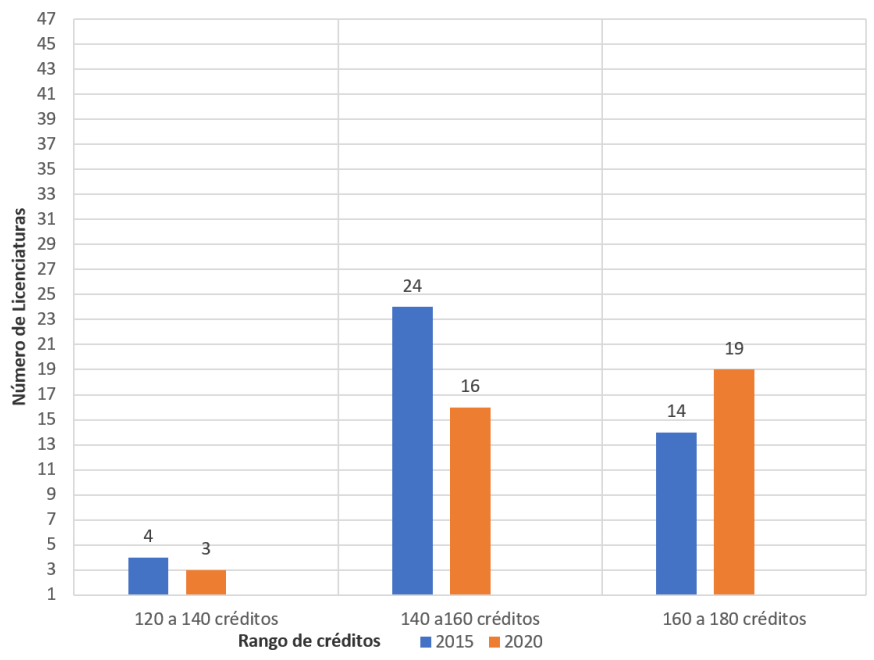

Fuente: creación propia.

En el análisis de los programas también se encuentra que la propuesta de las licenciaturas respecto a los créditos académicos varía en cierto grado (figura 2), puesto que en 2015 había cuatro programas que ofrecían una propuesta inferior a 140 créditos y en 2020 se registran tres; en 2015 veinticuatro programas tenían entre 141 y 160 créditos, en 2020 hay dieciséis; por último, en 2015 se ofrecían catorce licenciaturas de entre 161 y 180 créditos, y en 2020 son diecinueve. Por lo anterior, se evidencia un interés de los programas por ampliar su número de créditos debido a que la norma convoca a una mayor cantidad de tiempo para los procesos de investigación y de práctica. 


\section{Conclusiones}

Las propuestas curriculares de formación de profesores en humanidades y lengua castellana hacen un especial énfasis en el desarrollo humano y social de la persona basándose en criterios como la flexibilidad, la interdisciplinariedad, la integralidad y la transdisciplinariedad. Ello supone un desarrollo humanista que gira en torno a la construcción permanente, a partir de las relaciones que se establecen con la cultura y, por tanto, con el entorno social, lo que implica que el proceso de aprendizaje de los futuros educadores sea constante y se centre en las relaciones con los otros.

En esa medida, se evidencia que la mayoría de los programas responden a las directrices de las políticas nacionales en relación con los componentes propuestos, ya que la totalidad de las licenciaturas estructuran su programa en fundamentos generales, saberes específicos y disciplinares, pedagogía y ciencias de la educación, y didácticas específicas. Lo anterior se justifica, en términos generales, con el propósito de ofrecer una formación que integre la reflexión, la investigación y la innovación sobre los procesos de enseñanza y aprendizaje de la lengua castellana y diversas lenguas modernas.

Por consiguiente, se observa que todas las licenciaturas responden a los criterios curriculares propuestos por el Consejo Nacional de Acreditación (CNA) y el Ministerio de Educación Nacional (MEN), en cuanto a que los proyectos curriculares proponen una formación integral basada en el compromiso social y pedagógico. Estos se fundamentan, en primer lugar, en el principio de desarroIlar un proyecto educativo que posibilite la convivencia y facilite el aprendizaje por medio de la significación; en segundo lugar, por el cambio de denominación, puesto que todos los programas se ajustaron a la Resolución 02041 de 2016.

No obstante, se evidencia un cambio poco radical en la denominación de los programas, puesto que de los cuarenta y dos programas ofertados en 2015, veintiocho se enfocaban en la lengua 
castellana y catorce hacían énfasis en las lenguas o idiomas. En 2020, de las cuarenta y dos licenciaturas iniciales, nueve se habían dejado de ofertar, y además se crearon cinco nuevas de distintas instituciones, para un total de treinta y ocho programas; de estos, veintinueve optaron por la lengua castellana y nueve por las lenguas modernas o los idiomas extranjeros. Por lo tanto, cada institución ratificó su programa de formación en torno a la lengua; además de ello, cabe resaltar que dos instituciones crearon nuevos programas en la implementación de la resolución, por lo que ahora ofertan un programa para lengua castellana y otro para lenguas.

Respecto a la cantidad de créditos, pocos programas optaron por reducir el número de créditos académicos, y la mayoría amplió en esa medida su propuesta; por lo anterior, es notable la influencia de la norma, que convoca a una mayor cantidad de tiempo para los procesos de investigación y de práctica.

En consecuencia, los programas se justifican en una demanda social de profesionales en el área de las humanidades que orienten y mejoren los procesos de socialización y aprendizaje, pues estos son la base de la interacción del hombre como ser social y de su educación. Así, en espacios de interacción con comunidades, como el propiciado por las prácticas sociales y las profesionales, los futuros licenciados desarrollan procesos comunicativos.

Finalmente, los proyectos curriculares de los programas en humanidades y lengua castellana presentan las prácticas sociales y profesionales como un escenario de impacto social en el que los estudiantes tienen contacto con contextos educativos diversos y ponen en acción su propio proyecto de formación. 


\section{Referencias bibliográficas}

Baeza, M. (2002). De las metodologías cualitativas en investigación científico-social: diseño y uso de instrumentos en la producción de sentido. Concepción: Universidad de Concepción.

Gallardo, Y. y Moreno, A. (1999). Serie Aprender a investigar: módulo 3, recolección de la información. Bogotá: Icfes.

Garcés, D. (2008). Tensiones entre el desarrollo curricular del sistema educativo colombiano y el proceso de construcción cultural afrocolombiana. Artículo presentado en el II Foro Internacional sobre Educación Superior: Poblaciones Indígenas y Afrocolombianas, Bogotá, Colombia. Recuperado de http://www.colombiaaprende.edu.co/html/ mediateca/1607/articles-174672_archivo.pdf

Hernández, R., Fernández, C. y Baptista, P. (2006). Metodología de la investigación. Ciudad de México: McGraw Hill.

MEN (Ministerio de Educación Nacional). (2016). Resolución 02041: por la cual se establecen las características específicas de calidad de los programas de licenciatura para la obtención, renovación o modificación del registro calificado. Recuperado de https://www.mineducacion.gov. co/1759/w3-article-356144.html?_noredirect=1

Nicolescu, B. (2006). Transdiciplinariedad: pasado, presente y futuro. Primera parte. En: Revista Visión docente con-ciencia, (31): 15-31. http:// www.tercercongresomundialtransdisciplinariedad.mx/en/wp-content/ uploads/2019/08/Transdisciplinariedad-PASADO-PRESENTE-FUTURO-. pdf

Perdomo, W., López, L. y Mahecha, M. (2016). Panorama del lenguaje y la literatura en las licenciaturas en humanidades en Colombia. Revista Interamericana de Educación, Pedagogía y Estudios Culturales, 9(2), 3958. 
Perdomo, W., Buitrago, A. y Báez, C. (2017). El lenguaje en las humanidades: panorama de la formación de docentes en humanidades y lengua castellana. Bogotá: Corporación Universitaria Minuto de Dios.

Perdomo, W. y Buitrago, A. (2018). Lenguaje y currículo: una mirada al lenguaje en Colombia. Beau Bassin: Editorial Académica Española.

Reguera, A. (2008). Metodología en investigación lingüística. Buenos Aires: Brujas.

Rincón, G. (coord.) (2004). Marco de fundamentación conceptual y especificaciones del examen para las licenciaturas en Educación Básica con Énfasis en Humanidades y Lengua Castellana, Lengua Castellana y Literatura, y Lenguas Modernas. Cali: Universidad del Valle.

VV.AA. (2013). Lineamientos para la acreditación de programas de pregrado. Bogotá: Consejo Nacional de Acreditación CNA.

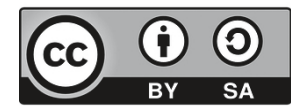

